\title{
PINTURAS INÉDITAS DEL SIGLO XVI: BENSON, PIETER COECKE Y EL MAESTRO DEL HIJO PRÓDIGO
}

\author{
POR \\ ELISA BERMEJO \\ Dpto. de $\mathrm{H}^{\mathrm{a}}$ del Arte. C.S.I.C.
}

There are identified four netherlandish pictures of A. Benson, P. Coecke and the Master of the Prodigal Son from spanish private collections.

No es muy frecuente poder presentar un grupo de obras de los Países Bajos, desconocidas hasta ahora, en las que concurren valores singulares independientes, por supuesto, de la época, la escuela y el estilo personal de cada uno de sus autores.

En primer lugar parece obligado aludir a la calidad que se aprecia en todas las pinturas, aquí estudiadas, sin olvidar el especial interés de cada una para un mejor conocimiento de los artistas que las pintaron puesto que del análisis, que exponemos, resultan conclusiones que perfilan o aclaran, en cada caso, algunas de las noticias documentales que nos han llegado, y las influencias estilísticas que en ellas se advierten.

Sin intentar establecer prioridades, entre estas obras, parece lo mas acertado presentarlas en orden cronológico teniendo en cuenta la fecha aproximada que. tras un detenido estudio, hemos estimado para cada una así como la escuela a la que corresponden por su estilo y caracteres.

\section{AMBROSIUS BENSON:}

Cristo Bendiciendo (T. 51,5 × 36 cms). Madrid. Colección particular. La tabla muestra a Cristo de busto, en posición frontal, con gesto de bendecir con la mano derecha y sosteniendo con la izquierda un globo de cristal cruzado por aros y con remate de cruz en simulada orfebrería dorada y con piedras preciosas en la decoración. Es la manera simbólica de representarle como Salvador del Mundo. Viste túnica y capa pluvial, en rojo, ornamentación bordada en oro y pedrería que cierra, al centro, por broche de diseño lobulado, con figurilla central, sentada en un trono representación probable del Padre Eterno o Todopoderoso. De la cabeza surgen dorados rayos de distinta longitud (Fig. 1).

Se trata de una composición iconográfica do larga tradición en los Países Bajos cultivada, con 


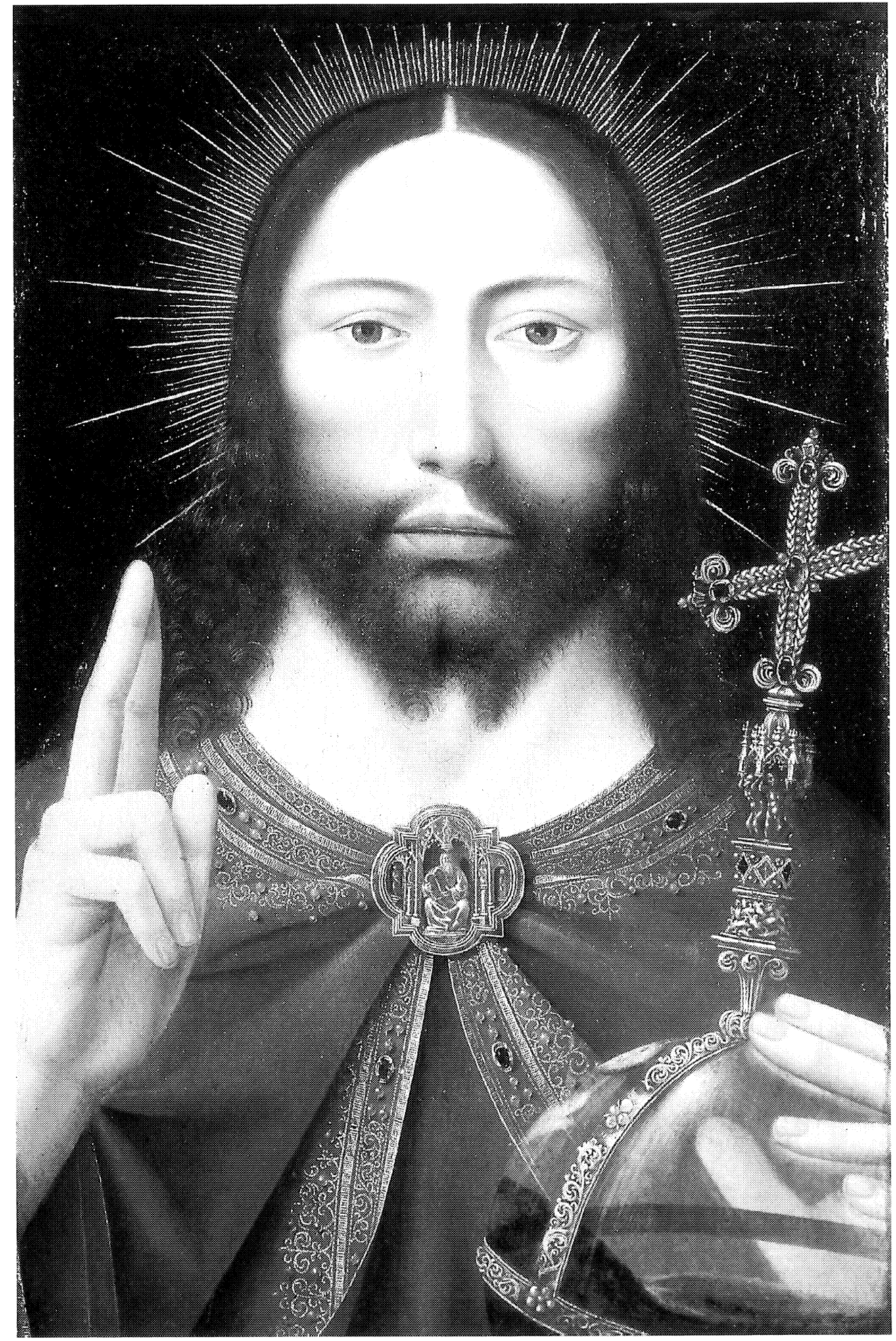

Fig. 1. AMBROSIUS BENSON: Cristo Salvador: Madrid. Colección particular 
ligeras variantes por pintores del siglo xv, de la importancia de Jan Van Eyck, que sigue vigente, durante el siglo XVI, en pinturas debidas a diversos artistas con las naturales diferencias de época, escuela y estilo, según los autores que las realizaron. Quizás una constante es representar a Cristo de busto o poco más, en posición absolutamente frontal y salvo en casos singulares, como en los ejemplares salidos de la mano de Dieric Bouts, su hijo Albert y sus respectivos talleres en los que prima cierto sentido dramático del dolor, lo más frecuente es presentar a Cristo con rostro de rasgos hieráticos, es decir, ausente de gestos que expresen sentimientos para dotar su figura de majestad divina.

Las variantes, a las que aludimos, se refieren a detalles complementarios que sirven para denominar las representaciones de Cristo. Los primeros ejemplares atribuidos a Jan van Eyck y seguidores se distinguen por mostrar solamente el rostro, rodeado por rayos de oro, y una mínima parte de la túnica. Friedländer opina, junto a otros autores, sin unanimidad, que el original de Van Eyck es el que se conserva en el Museo de Berlín ${ }^{1}$ (Fig. 2).

Otra versión del tema se debe, como queda dicho, a Dieric y Albert Bouts, pero ambos artistas, al igual que sus talleres e imitadores, se caracterizan por la expresión dolorida del rostro de Cristo ${ }^{2}$.

J. Lavalleye dio a conocer, en 1953, un ejemplar en colección particular española de mano de Gerard David, que representa a Cristo mostrando las llagas de sus manos ${ }^{3}$. Está realizado con igual tipo de iluminación que el aquí estudiado y con la mirada dirigida al espectador (Fig. 3). Otro ejemplar atribuido por Friedländer al propio David, que se conserva en la colección Johnson de Filadelfia, lo muestra con la mano derecha en actitud de bendecir 4 .

Quentin Metsys es autor de un espléndido ejemplar, que forma pareja con una Virgen en oración, en el que presenta a Cristo como Salvador del Mundo bendiciendo con una mano y apoyada la otra en un globo rematado por hermosa cruz de orfebrería ${ }^{5}$. Es, precisamente, este gran pintor de la escuela de Amberes el que consigue una mayor difusión de este tipo iconográfico a juzgar por las numerosas réplicas e imitaciones que salen, en su época, realizadas por diversos pintores de los Paises Bajos (Fig. 4).

Es por tanto indudable que se trata de una imagen de devoción que obtuvo una gran acogida entre la clientela de buen número de artistas y Ambrosius Benson, es uno de ellos. Este pintor, de origen lombardo, desarrolla en Brujas toda su carrera artística conocida y, en esta ciudad muere en 1550. También se sabe que, en ella, trabajó, un tiempo, como colaborador de Gerard David. En la tabla de colección madrileña, que presentamos por vez primera, hay como una síntesis de las dos principales composiciones citadas. Participa del hieratismo que le proporciona, el rostro frontal de ojos abiertos y mirada enigmática utilizado por Van Eyck y Gerard David y se suma a la idea simbólica de mostrarle como Salvador, bendiciendo y con el globo crucífero que valora, ante todo, su papel redentor.

En, 1953 publicó el profesor Lavalleye por primera vez un hermoso cuadro que, con acierto, considera realizado por Ambrosius Benson en el que aparece Cristo bendiciendo y sosteniendo el

\footnotetext{
1 Max J. Friedländer: Early Netherländish Painting. The Van Eycks-Petrus Christas, vol. I. Comentarios y notas N. Veronee-Verhaegen. Leyden. Bruselas, 1967, págs. 69-70, lám. 63. E. Bermejo: La Pintura de los Primitivos Flamencos en España, vol. I, Madrid, 1980, núm. 10, págs. 58-59, fig. 24, dimos a conocer un ejemplar próximo al de Berlín.

2 E. Bermejo: La Pintura do los Primitivos Flamencos en España, vol. II. Madrid, 1982, figs. 25-36 y 40-54.

3 J. Lavalleye: Les Primitifs Flamands, II Repertoire des peintures flamandes des quinzième et seizième siècles. "Collections d’Espagne», I. Amberes, 1953, núm. 2, pág. 9, lám. II. Salió de España y se recoge como adición en el vol. VI b de M. J. (Friedländer: Early Netheslandish Painting. Hans Memling and Gerard David. Comentarios y notas N. Veronee. Verhaegen. Leyden. Bruselas, 1971. Add 297, pág. 117, lám. 269, donde se encuentra actualmente (Greenville, South Carolina. The Bob Jones University. Collection of Religious Paintings. Cat n. 120; T. 47,8 × 35,4 cm.).

${ }_{4}^{4}$ Max J. Friedländer: Early..., vol. VI b, 1971, núm. 200, lám. 205.

5 Ibidem, vol. VII, Quentin Massys. Comentarios y notas H. Pauwels. Leyden. Bruselas, 1971, núm. 5, pág. 60, lám. 12.
} 

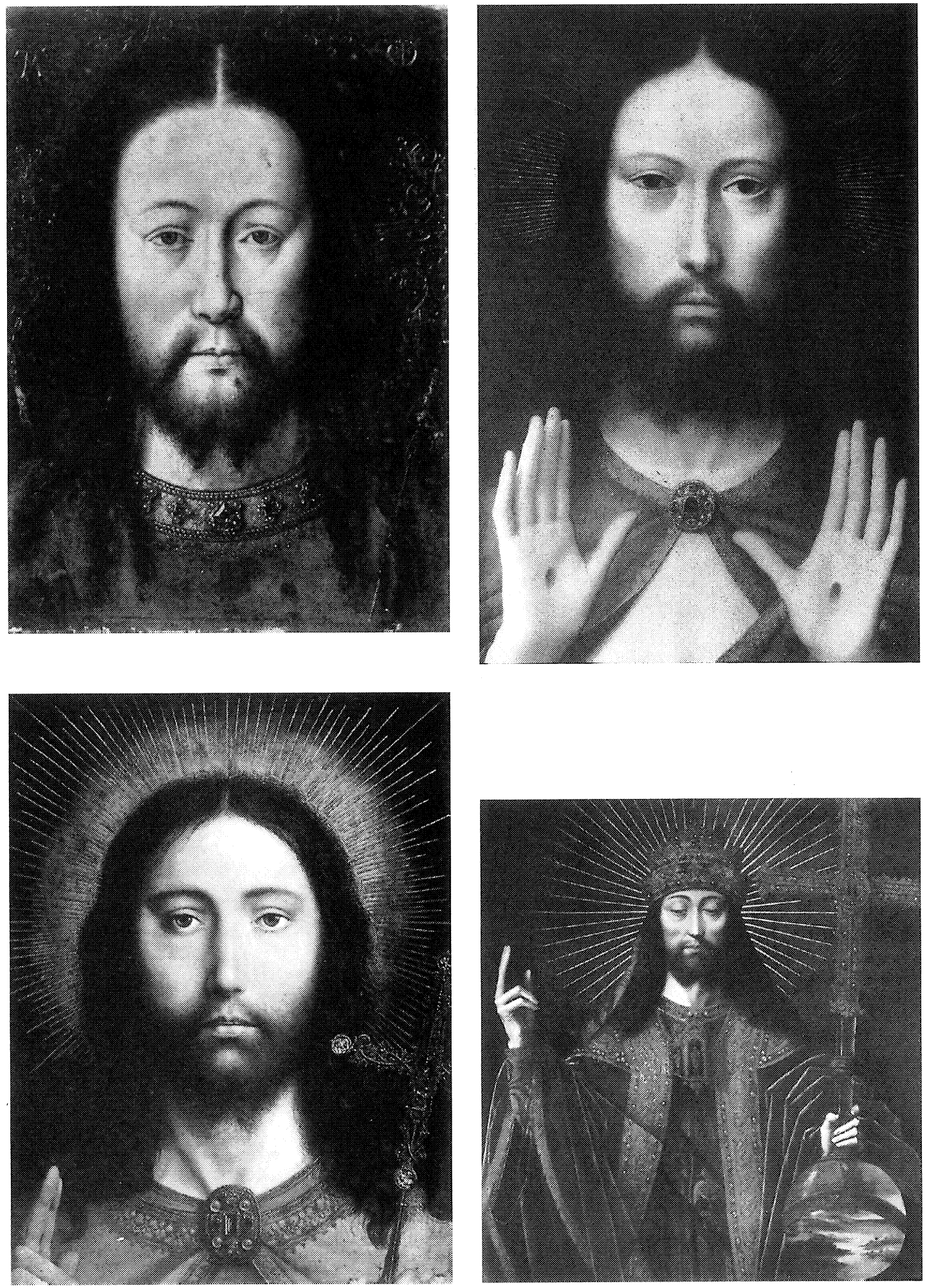

Fig. 2. J. Van Eyck.: Cabeza de Cristo. Berlín. Museo. - Fig. 3. G. David: Cristo mostrando las llagas.Greenville. South Carolina. The Bob Jones University. - Fig. 4. Q. Metsys: Cristo Salvador. Amberes. Musée Royal des Beaux-Arts. - Fig. 5. A Benson: Cristo Salvador. Madrid. Ant. col. Traumann. 
globo del mundo pero, el Salvador, no esta representado de busto como es lo habitual, sino de medio cuerpo. Se muestra, además, con los párpados caídos y mira hacia abajo ${ }^{6}$ (Fig. 6). Estos, y otros detalles que añadiremos, nos sirven para situar la tabla. motivo de este trabajo, en la fecha probable de su realización. Hemos de señalar, no obstante, que los caracteres más típicos de Benson se muestran con igual evidencia en ambas pinturas. Es el caso de las manos grandes, de dedos largos y falanges medias que acusan los nudillos, los ojos bridados, cejas rectas y modelado a base de luces y sombras que marcan los pómulos y se distribuyen en forma difuminada, posible recuerdo de su primera formación lombarda.

En la pintura publicada por Lavalleye se pueden advertir detalles que no aparecen en la nuestra como pueden ser la túnica adamascada. la mayor importancia concedida a la decoración da la capa pluvial, el estar cubierto con una tiara y sobre todo, es muy significativo, un detalle de carácter típicamente bensoniano como es la serie de lineas blancas que subrayan los pliegues de la tela técnica que emplea Benson en las obras de avanzada época para conseguir el efecto y calidad del terciopelo. Por ello aludimos. poco mas arriba, a la importancia de estas diferencias para intentar fijar una fecha, aproximada. a esta nueva obra de Benson que, a su fina calidad, añade la de ser una composición única en su producción, como puede comprobarse al repasar la excelente monografía de Georges Marlier sobre este interesante pintor ${ }^{7}$. Según la evolución artística que el propio Marlier establece, en su publicación, y atendiendo a los caracteres que mencionamos parece probable que el cuadro de colección madrileña se pintara por los años 1530-1532.

\section{PIETER COECKE:}

\section{Adoración de los Magos}

Se trata de una Adoración de los Magos (Barcelona, Comercio de Arte, T. 105,5 x $69 \mathrm{~cm}$ ) en la que el remate superior lobulado, de la tabla, parece indicar que debió de ser centro de un tríptico del que, como en tantos casos. desconocemos la posible existencia de puertas. Creemos es una obra con 1a calidad y belleza suficientes para dedicarle un análisis estilístico a efectos de puntualizar la existencia de elementos compositivos de interés y 1a probable fecha de su realización (Fig. 6).

Esta Adoración de los Magos tiene como escenario un espacio en el que los protagonistas aparecen, en primer plano, sobre un suelo campestre como se aprecia, en el borde inferior, por las hierbas, la piedra del ángulo derecho y los pequeños cantos rodados, que lo salpican, típicos de Coecke y, como es frecuente, en la interpretación flamenca del tema, se enriquece con un fondo de arquitectura que representa las ruinas de un templo, con elementos constructivos y decorativos plenamente renacentistas que abre, a través de los restos de un amplio arco de medio punto, a un paisaje con edificios y menudos personajes que lo animan y crean sentido de profundidad.

El grupo principal esta compuesto por el Mago arrodillado, la Virgen con el Niño y San José, en pie tras ellos, lo que Georges Marlier denomina composición en triángulo y una de las primeras de la pintura flamenca ${ }^{8}$ que viene de Jan Van Dornicke, pintor de Amberes y suegro de Pieter Coecke del que éste repitió varias composiciones. El centro del tríptico, de Dornicke, de colección particular de Paris (Fig. 7) lo sigue Coecke, puntualmente, en la tabla central de la Adoración de los Magos, del Prado (n 2223;87 x $35 \mathrm{~cm}$ ) en la que Marlier advierte el contraste

\footnotetext{
6 J. Lavalleye: Les Primitifs Flamands, II. Repertoire des peintures flamandes des quinzième et seizième siècles. "Collections d'Espagne», I. Amberes, 1953, núm. 3, pág. 10, lám. III.

7 G. Marlier: Ambrosius Benson et la peinture a Bruges au temps de Charles Quint. Bruselas, 1957.

8 G. Marlier: La Renaissance Flamande Pierre Coeck d'Alost. Bruselas, 1966, pág. 126.
} 


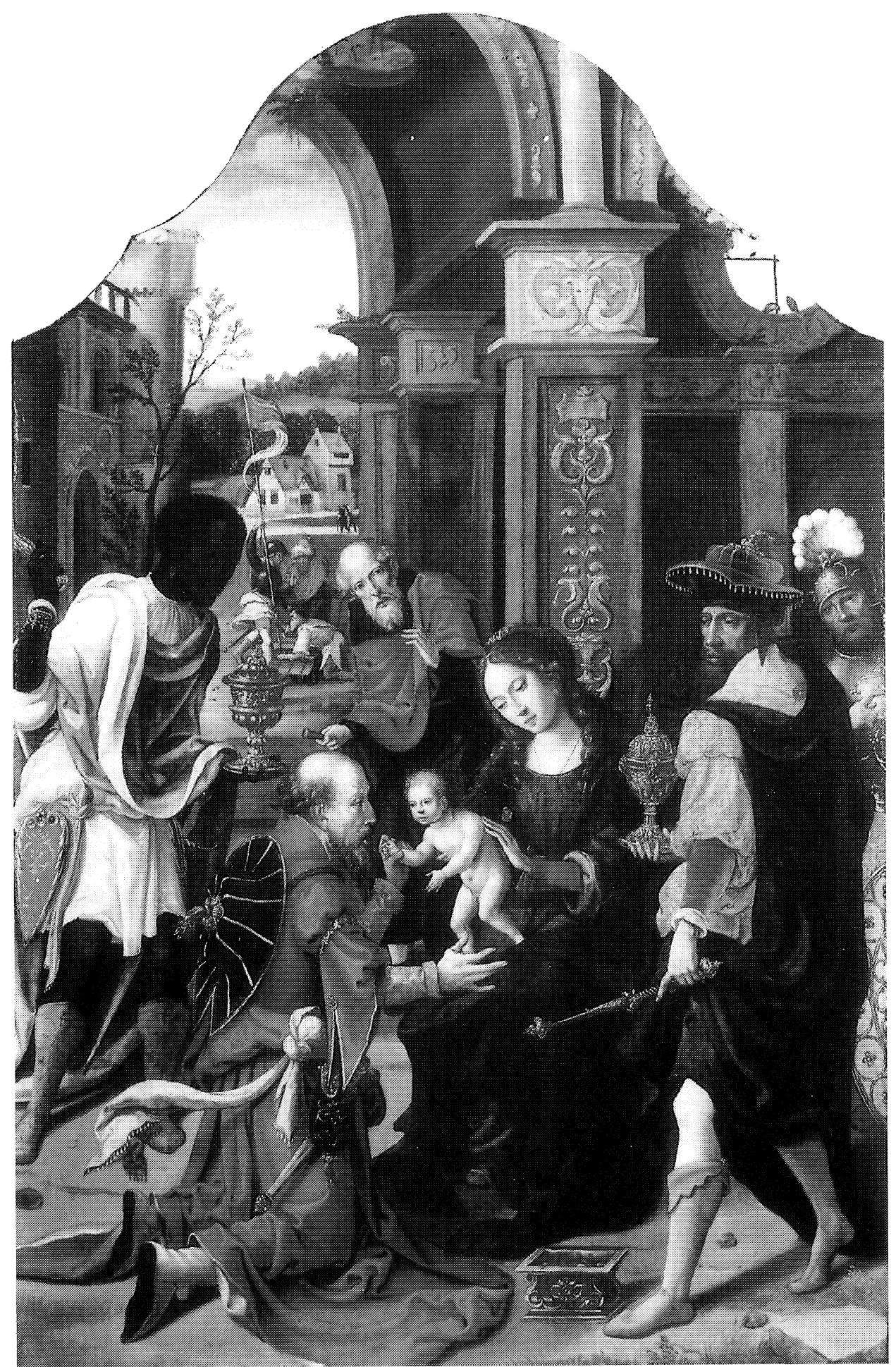

Fig. 6. P. Coecke: Adoración de los Magos. Barcelona. Col. particular. 
con las otras versiones que no considera de su mano (Fig. 8). La tabla de Barcelona mantiene, para el grupo principal, el recuerdo del esquema triangular aunque, al incluir a los tres Magos en la misma escena (tanto en el tríptico de París como en el del Prado, dos de ellos figuran en las puertas), varía su aspecto general y crea una presentación nueva que muestra. al tiempo, un escalonamiento diagonal que va del personaje vestido de guerrero, con casco y penacho de plumas al Mago arrodillado. A propósito del guerrero, es curioso señalar que Marlier, del que aparece en el segundo plano izquierdo del Prado, dice que «tiene los rasgos del retrato, grabado, de Pieter Coecke» ${ }^{9}$ que, en la tabla de Barcelona, se muestran semejantes y quizás mas evidentes. Sin conceder al detalle valor decisivo apuntamos que el gesto de su mano con el índice señalándose a sí mismo podría aludir a este extremo.

La Virgen con el Niño aparece sentada ante un pilar como en las dos citadas. de colección particular parisina, de Dornicke y la del Prado de Coecke pero sin el velo, de aspecto más tradicional, que cubre sus cabezas, sino luciendo, aquí, un peinado con nuevo y coqueto tocado. En cambio, San José muestra actitud y vestiduras parecidas en estos tres ejemplares. Por otra parte, el tierno gesto del Mago arrodillado tomando, con su mano izquierda, una manecita del Niño lo encontramos, también, en el centro del tríptico de la Adoración de los Magos, de medio cuerpo, del Museo de la Fundación Lázaro Galdiano de Madrid, considerada por Marlier «como obra maestra de Jan Van Dornicke» aunque, con la ligera variante de que. en esta última, la retiene con ambas manos ${ }^{10}$ (Fig. 9). El Mago negro se presenta de aspecto y actitud casi idénticos en la Adoración de la Galería Robert Frunck, de Bruselas, de mano de Van Dormicke ${ }^{11}$ y en los ejemplares de Coecke del Country Museum of Art, ${ }^{12}$ de los Angeles y de la Universidad de Princeton ${ }^{13}$ aunque en la tabla que estudiamos, se sitúa a la izquierda y no a la derecha como en los ejemplares citados. El rey Melchor, del lateral derecho de nuestra tabla, se presenta en actitud muy similar al de la Adoración de los Magos, de Coecke de la colección real inglesa que se conserva en el Castillo de Hampton Court (n 268 T. 48,5 x 38 cm) (Fig. 10) en la que Marlier ${ }^{14}$ advierte que el personaje "avanza con paso decidido y ligero" es decir, con idéntica postura, al apoyar los pies sobre el suelo lo que marca, con claridad, el paso del llamado «manierismo prerrenaciente al romanismo». Esto no impide que, como hemos apuntado. algún personaje proceda aun del repertorio de Jan Van Dormicke pero, los caracteres más personales de Coecke: una composición más elaborada, mayor movimiento en las actitudes y el aspecto general de los elementos decorativos, se ven próximos al estilo italiano de la época.

Entendemos que, es arriesgado fijar fechas, demasiado precisas. para las pinturas de Pieter Coecke pero habida cuenta de que Marlier sitúa la Adoración del Prado (n 2223) hacia 1530 y el apreciable avance en novedades compositivas, del cuadro de Barcelona, nos permite considerar que Coecke debió de pintarlo justo antes de su partida para Constantinopla o en el momento de su vuelta para Amberes. es decir ca.1533-1535.

Añade interés a la pintura el hecho de que no se conserva, que sepamos, ningún otro ejemplar, de Pieter Coecke. con igual composición, lo que elimina el carácter de réplica y como su finura de calidad hace impensable intervención alguna del taller, nos permite incluir esta nueva Adoración de los Magos entre las mejores salidas de la propia mano del pintor.

\footnotetext{
9 Ibidem, pág. 128

10 Ibidem, pág. 155, fig. 67.

11 Ibidem, fig. 41.

12 Ibidem, fig. 47.

13 Ibidem, pág. 49

14 Ibidem, pág. 171, fig. 112.
} 

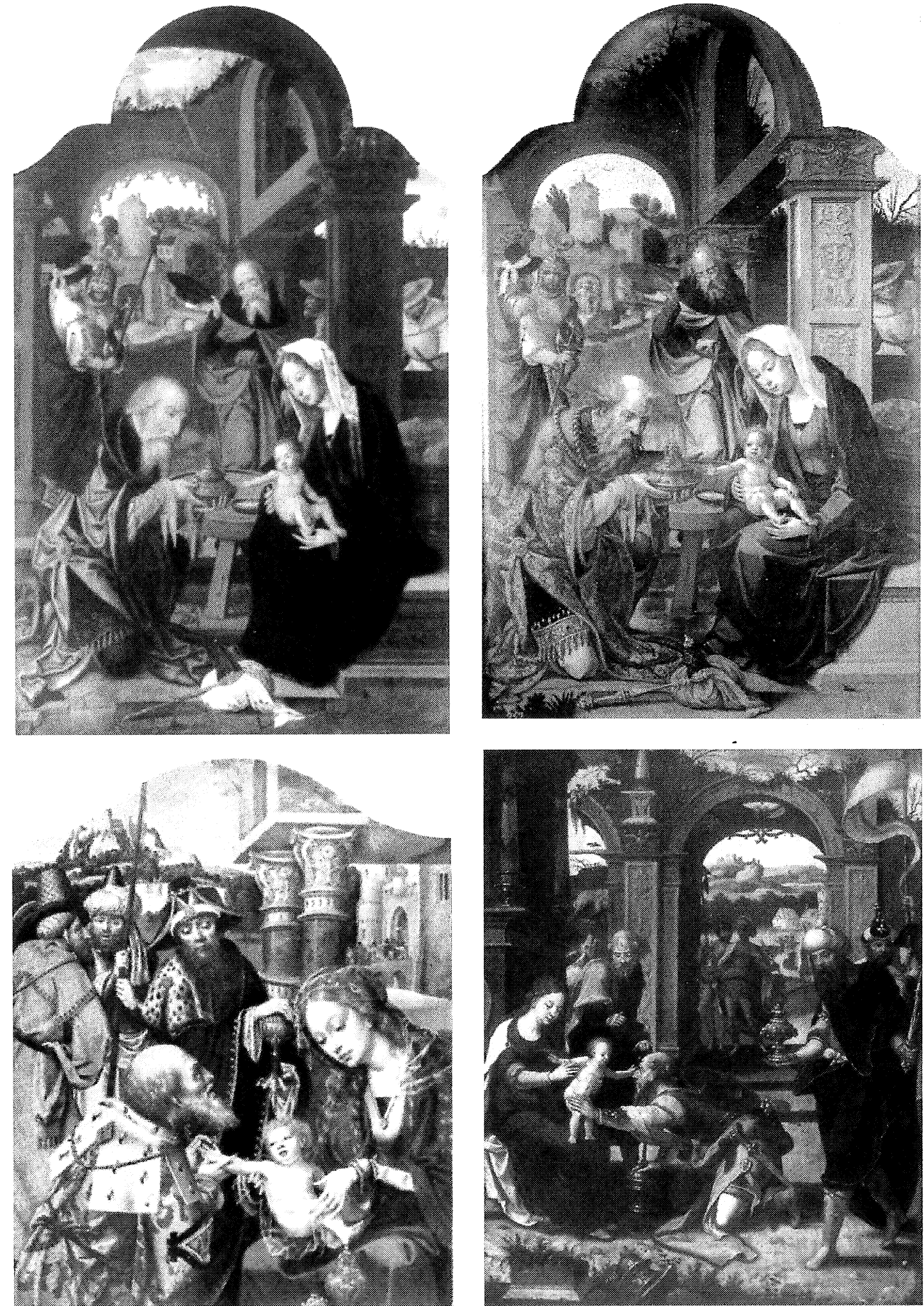

Fig 7 J Van Dornicke: Adoración de los Magos. París. colección particular. - Fig. 8. P. Coecke: Adoración de los Magos. Madrid. Museo del Prado. - Fig. 9. J. Van Dornicke: Adoración de los Magos. Madrid. Museo Fundación Lázaro Galdiano. - Fig. 10. P. Coecke: Adoración de los Magos. Hampton Court. Colección Real inglesa. 


\section{Nacimiento de la Virgen}

En el Nacimiento de la Virgen (Madrid, colección particular, T 82.5 x $65 \mathrm{~cm}$ ) concurren razones para considerarlo pieza importante, dentro del catálogo de obras del pintor Pieter Coecke d'Alost, como intentamos probar, seguidamente, a través de un estudio estilístico del cuadro con apoyo de argumentos basados en los datos conocidos de la vida y de la obra de este fecundo y brillante artista de Amberes (Fig. 11).

El aspecto actual de la tabla permite presumir que pudo formar parte de un conjunto, más amplio, dedicado a la Vida de la Virgen y aún quizás que, en su origen, sus dimensiones fueran algo superiores ya que en los bordes, sobre todo en el de arriba, aparecen inacabados elementos importantes que desequilibran. un tanto, la composición.

En primer lugar, conviene describir la escena para rastrear, en ella, relaciones o influencias que, junto a su composición, caracteres de estilo y color sirvan para vincular este Nacimiento, al arte de Pieter Coecke. El escenario presenta una disposición, de armoniosa flexibilidad, en tres planos, perfectamente diferenciados, compuestos con un sentido que refleja, de una parte, su relación con Bernard Van Orley y, de otra, su cabal conocimiento de las novedades aportadas por el clasicismo italiano, con criterio de la jerarquía de valores no sólo por la propia disposición de los personajes, en la escena, sino por el papel que desempeñan frente al espectador, aunque sin olvidar el carácter narrativo, de larga tradición flamenca, como atestigua la inclusión, al fondo, del Abrazo de San Joaquin y Santa Ana.

Como queda dicho. vamos a recordar algunas de las noticias, conocidas, sobre la vida de Pieter Coecke para reforzar los dos argumentos principales que sirven, a nuestro propósito, de identificar el Nacimiento de la Virgen como pintura realizada de la propia mano de este pintor. Los documentos conservados dan cuenta de que la mayor parte de la carrera artística de Coecke transcurrió en Amberes en donde fue recibido maestro en 1527. El hecho de que antes de esa fecha, no aparezca inscrito como discípulo permite suponer que hiciera su aprendizaje fuera de esta ciudad, probablemente, en Bruselas ${ }^{15}$. Al respecto no existe documento probatorio pero como opina Georges Marlier, excelente biógrafo de Coecke, hemos de creer la palabra del historiador de la pintura flamenca Carel van Mandar, quien en la edición de 1604 de su «Het Schilder Boeck» en traducción íntegra y literal ${ }^{16}$ dice: ... Fue también pintor de la Majestad Imperial Carli Quinti al servicio del cual murió en Amberes... en el año del Señor 1550" Van Mander asegura también que el joven Coecke aprendió su oficio con «Bernard de Bruxelles», es decir, con Bernard van Orley y que hizo un viaje a Italia.

Aún a falta de base documental su presunto aprendizaje con el conocido maestro bruselense tiene su justificación en varias obras atribuidas, con todo derecho. a Coecke porque revelan la clara influencia de Van Orley, como en el caso de la aquí estudiada. La estancia del pintor en Italia, tampoco puede confirmarse por datos de archivo pero, citando de nuevo a Marlier 17: “... no podría ponerse en duda. Sólo en Italia pudo adquirir el suficiente conocimiento de la lengua para traducir una obra tan técnica como los libros de Arquitectura de Sebastián Serlio". Por otra parte. la estrecha relación con Van Orley, que debió de mantener a lo largo de su vida, parece confirmarla el hecho, si conocido por documentos de archivo, de que «... cuando murió P. Coecke fue a reunirse en la iglesia de Saint-Gery, de Bruselas, con su presunto maestro» ${ }^{18}$.

Conviene ahora, comparar el Nacimiento de la Virgen, en, colección madrileña, de Coecke con el mismo tema pintado en una puerta, que tiene su pareja; muestra la izquierda, en el reverso,

15 Ibidem, pág. 38.

16 Ibidem, págs. 26-27.

17 Ibidem, pág. 27.

18 Ibidem, pág. 35. 


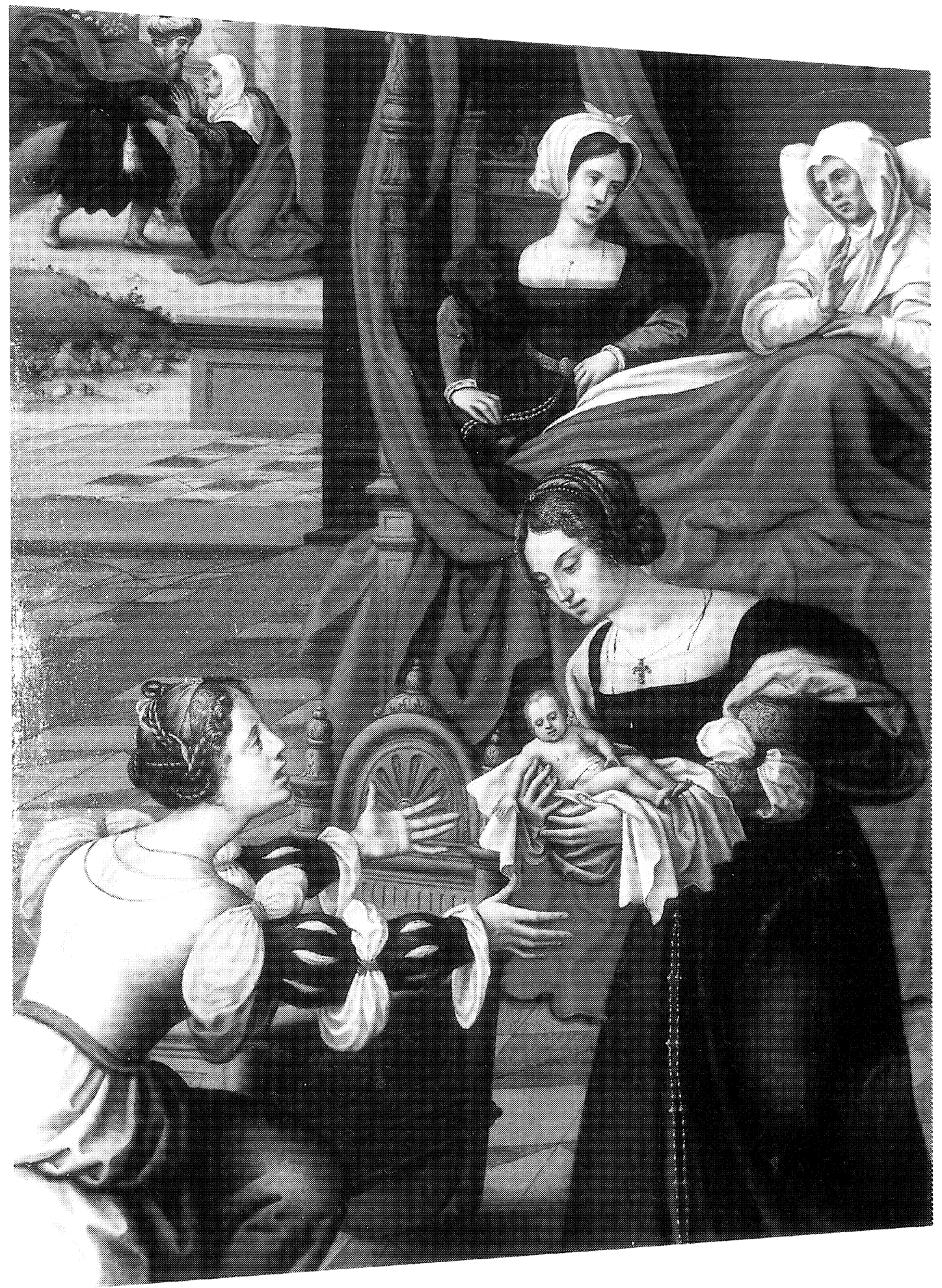

Fig. 11. P. Coecke: Nacimiento de la Virgen. Madrid. Colección particular. 
los Desposorios de San Joaquín y Santa Ana y las dos escenas, de la puerta derecha, narran otras tantas historias de la Leyenda de Santa Ana que Friedländer atribuye a Van Orley ${ }^{19}$. La tabla con los Desposorios, lleva la fecha de 1528, es decir, a situar en el periodo avanzado de la carrera artística del maestro bruselense (1484-1441) (Figs. 12 y 13).

A la vista de los Nacimientos de la Virgen de ambos pintores, se pueden establecer una serie de analogías y diferencias, bien ilustrativas, para determinar, por una parte, la huella innegable del arte de Van Orley en Coecke que se advierte. sobre todo, en la composición pero, también, en detalles puntuales a los que aludiremos. El asunto principal lo componen igual número de personajes y su situación responde a un criterio similar. En primer término las dos figuras femeninas que atienden a la Virgen Niña y, en segundo plano, el importante lecho en el que reposa Santa Ana acompañada por una joven servidora y, al fondo, una abertura al exterior, con personajes a menor escala. Todo ello con semejanzas y variantes, del mayor interés, que permiten percibir, sin duda, el recuerdo de Van Orley junto con aportaciones romanistas de inspiración rafaelesca y caracteres absolutamente típicos del estilo de Coecke.

Entre estos cuadros de Orley y Coecke existen notables variantes pero están ralizados con concepto y sentido similares. Las mujeres, que ocupan el primer plano, aunque en actitudes invertidas presentan, en Bruselas, a la de la derecha alargando sus brazos para recoger a la recién nacida mientras que, en Madrid, es la de la izquierda la que los extiende para recibirla y acostarla en una cuna que tiene junto a ella. En cambio, las que alargan los brazos. en las dos tablas, aparecen de riguroso perfil y las otras dos giran, ligeramente. el rostro hacia el frente. También se invierte la disposición del lecho con Santa Ana y la joven acompañante: a la izquierda, con espacio, abierto al fondo derecha en Bruselas y a la inversa, en Madrid. Por otra parte, la Santa aparece corno adormecida, en la primera, mientras que la de la colección madrileña muestra la cabeza más erguida, vuelta hacia la joven que está a su lado y con un movimiento de manos que le añaden viveza. Se puede advertir que, en ambas, la Santa va totalmente vestida de blanco y apoya sobre sábanas y almohadas de igual color en contraste con el rojo del cubrecama. La ayudante muestra, en cada una. actitud diversa pero, curiosamente, el pañuelo de cabeza se anuda, en el centro por las puntas, de forma idéntica. Otra referencia análoga son los bullones o rollos de lienzo de tono blanco o muy claro que adorna las mangas de los trajes femeninos del primer término. Es interesante comparar la diferencia de tratar el plegado de las telas visible. sobre todo, en las que cubren el lecho tanto en su disposición como en el modelado que es mucho más amplio, con más flexibilidad, volumen y movimiento en el ejemplar madrileño y más apretado menudo y tendencia a lo vertical. en las caídas colgantes, en el del Museo bruselense lo que responde, sin duda, al diferente estilo y sensibilidad de los pintores de cada tabla. Igual tipo de plegado emplea Coecke en su José y la mujer de Putifar así como en la manera de pasar el colgante o caida alrededor de la columna del lecho ${ }^{20}$.

Es preciso, también, señalar lo que la pintura madrileña revela respecto a coincidencias con los caracteres reconocidos como típicos de Pieter Coecke y para ello, nada mejor que relacionarlos con los que aparecen en otras obras consideradas como suyas por los mas expertos estudiosos de su arte.

Antes conviene dejar constancia de que, como es sabido, la labor de Coecke comienza en el taller antuerpiano de su suegro Jan von Dormicke. alias "Maestro de 1518" y que durante no pocos años realiza numerosos ejemplares en los que, composiciones y tipos continúan repitiendo muchos de sus modelos. En cambio, los cuadros que corresponden ya a lo que Marlier denomina paso del Manierismo prerrenacentista al romanismo» ${ }^{21}$ se liberan de esa dependencia y se afir-

19 Max J. Friedländer: Early Netherlandish Painting. Gossaert, Van Orley, vol. VIII. Comentarios y notas H. Pauwels Leydem. Bruselas, 1972, núm. 93, págs. 103-104. ${ }^{20}$ G. Marlier: La Renaissance Flamande, pág. 286 , fig 226.

21 Ibidem, pág. 164. 

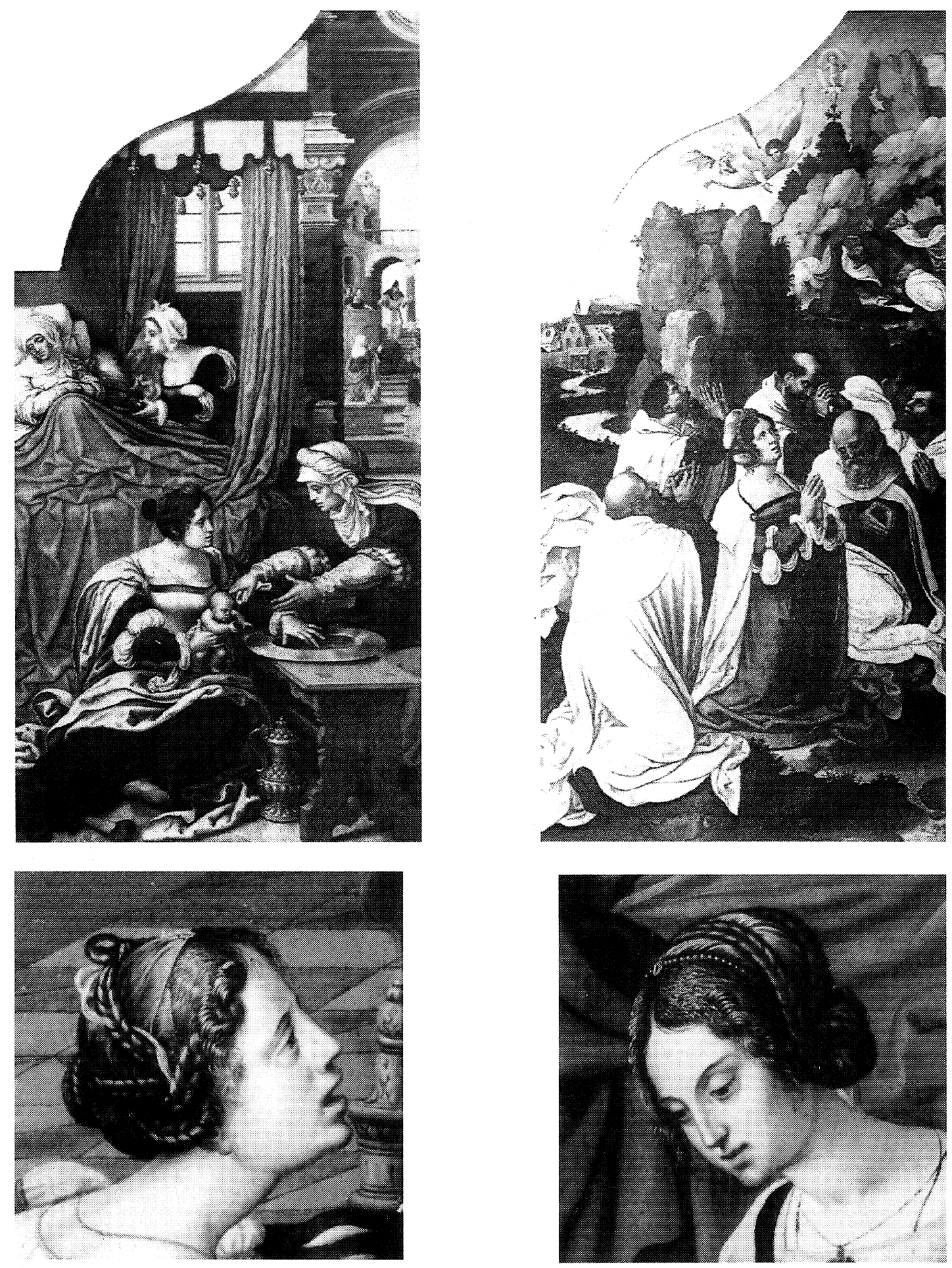

Figs. 12 y 13. B. Van Orley: Puertas. Leyenda de Santa Ana. Bruselas. Musees Royaux des Beaux Arts. Figs. 14 y $15 . \quad$ P. Coecke. Detalles fig. 11. 
man los caracteres más típicos del pintor. Las composiciones adquieren ritmo y movimientos nuevos que se aproximan al clasicismo italiano lo que se percibe incluso en la ornamentación. al abandonar la profusión decorativa de etapas anteriores.

El ideal femenino, de Pieter Coecke se manifiesta ante todo, en los rostros y, en esta época, en la que ha dejado atrás el recuerdo de los modelos de Jan Van Dormicke, se caracterizan por detalles muy personales que los hacen inconfundibles. El corte de óvalo adquiere una perfecta regularidad que lo aproxima al espíritu clásico, con boca de labios pequeños, bien delineados, un típico hoyuelo que puntúa el mentón y las mejillas avivadas por un suave toque rosado.

Los cabellos se recogen con femenina gracia por medio de bucles y trenzados tratados con la calidad de un orfebre y, para darles brillo, los aclara con reflejos dorados. es decir. tal como aparecen en las bellas jóvenes que ocupan el primer término de la tabla madrileña y que tienen el toque del cinquecento de Rafael y Garofalo. Característica también, es la trenza que a modo de diadema, les cruza la cabeza (Figs. 14 y 15). Ejemplos de estos rostros y peinados pueden verse también en otras pinturas de Coecke como la Sagrada Familia de la Colección Mouton de Bruselas ${ }^{22}$ de la que Marlier dice que «nos muestra a Coecke en la cima de su arte» o la Lucrecia, de la colección del Dr. André Matthey, de Lindau ${ }^{23}$ en la que figuran tanto los rasgos característicos, que señalamos para los rostros como «la cabellera mantenida por una trenza» que hace decir a Marlier: «todo proclama la paternidad de Pieter Coecke».

Otro rasgo que atrae nuestra atención es la actitud de la jóven del ángulo izquierdo de la tabla de Madrid, con una pierna doblada en movimiento de avance, levantando la cabeza, de riguroso perfil, con la boca entreabierta y cuello de delicadas curvas que, Coecke, hizo semejante en la Virgen, del Descanso en la Huida a Egipto (Viena Kunst Historisches Museum) donde luce, también un peinado muy similar que, para Marlier. parece tomado de Rafael ${ }^{24}$. Recoge, el propio historiador, las opiniones de Otto Benesch y Paul Wescher que mantenían ciertas reservas a que la pintura de Viena fuese, en su totalidad, de mano de Pieter Coecke pero añade su opinión personal que creemos muy acertada y es que. deja muy claro que el tríptico de la Crucifixión de Lisboa (Museo de Arte Antiga n. ${ }^{\circ}$ 12. Catálogo de 1951 n. ${ }^{\circ}$ 202) autentificado por documento descubierto por Leo Van Puyvelde ${ }^{25}$, en la Academia de Bellas Artes de Lisboa, era obra desconocida para los citados autores indecisos y. sin embargo, sirve de confirmación para la manera de ver el perfil de la Virgen del museo vienes. Por otra parte, para nuestra intención, si se compara la cabeza y el gesto de la joven en pie, a la derecha de la Crucifixión lisboeta, con la que comentamos en nuestra pintura, llegamos a idénticas conclusiones como demuestra la representación, en detalle, que publicamos (Figs. 16 y 17).

Tras el pormenorizado análisis expuesto a propósito del Nacimiento de la Virgen de colección particular de Madrid, inédito hasta ahora, nos parece lógico identificar su pintura con el estilo y manera de hacer habituales en Pieter Coecke por los años entre 1540 y 1545 que son los que apuntamos como fecha de su ejecución.

\footnotetext{
22 Ibidem, págs. 222-226, lám. color y fig. 162.

23 Ibidem, pág. 231, fig. 196.

24 Ibidem, pág. 270, fig. 214.

25 Ibidem, págs. 76-78.
} 


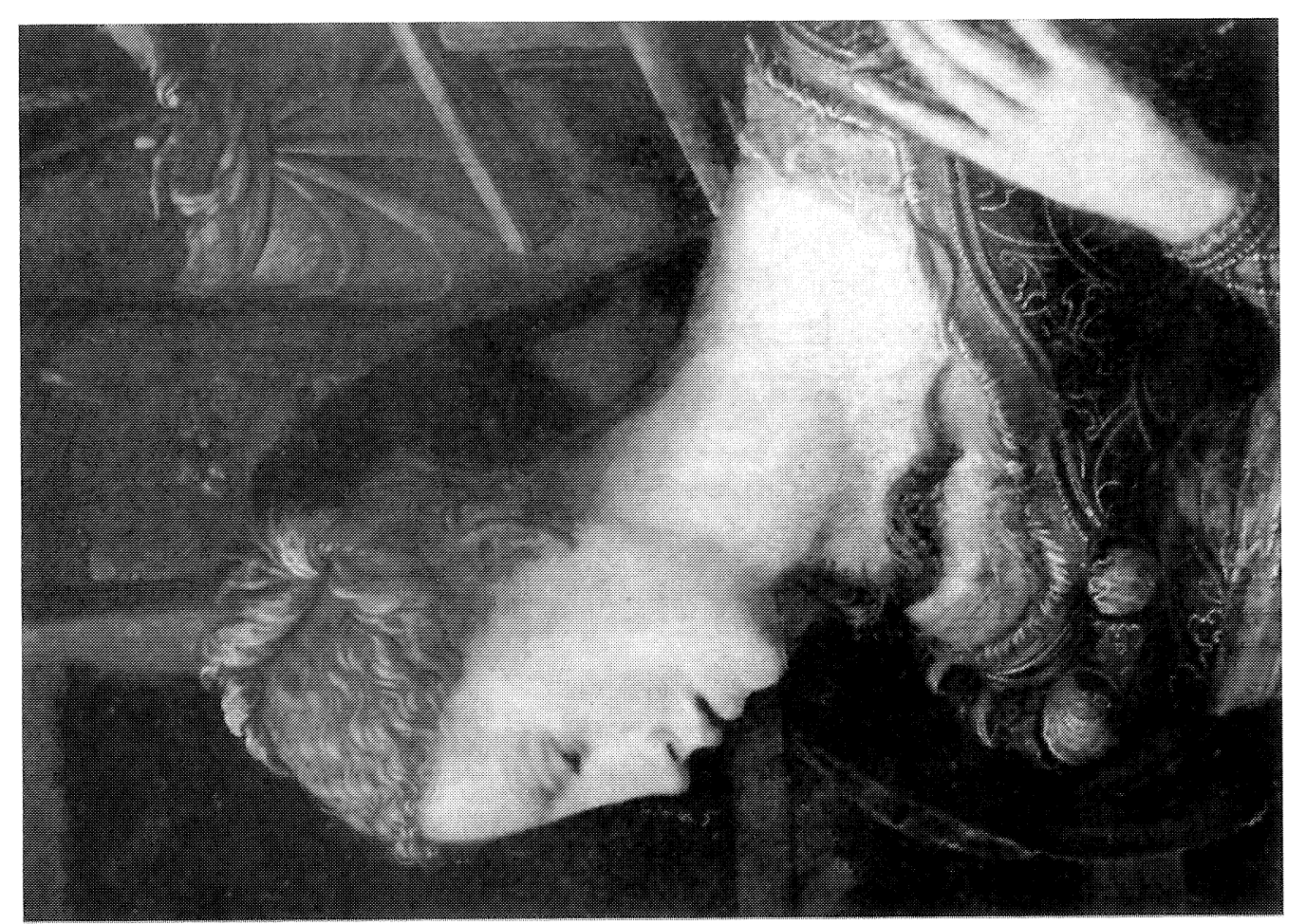

兽 


\section{MAESTRO DEL HIJO PRÓDIGO}

Virgen con el Niño, acompañada por dos personajes (Madrid, Colección particular, T. $96 \times$ $67 \mathrm{~cm}$.).

Esta bellísima pintura muestra una finura técnica y cromática que permite catalogarla como una de las mejores, entre las que conocemos, debidas al llamado «Maestro del Hijo Pródigo». Un análisis detenido de sus elementos compositivos formales, y de estilo nos servirá para establecer los fundamentos en los que se apoya nuestra identificación (Fig. 18).

Comenzamos por recordar que el nombre con el que se conoce al pintor, se lo dio, en 1909, el conocido historiador, de la pintura flamenca, G. Hulin de Loo ${ }^{26}$ quien al estudiar un grupo de obras estilísticamente homogéneo tomó, como referencia. el cuadro con la escena de El Hijo Pródigo en galante compañia (Viena, Kunsthistorisches Museum n T X cm), ${ }^{27}$ que por el momento, sigue vigente para catalogar los cuadros considerados como de propia mano del pintor y aún para reunir otros de taller que, por mostrar un estilo similar, aunque de inferior calidad, se suponen realizados por ayudantes o seguidores.

En 1923, Grete Ring, continúa el estudio sobre el Maestro 28 refutando anteriores sugerencias de identificación emitidas por algunos autores, que cita, y proponiendo. por su parte, otra diferente desechada, a su vez, por F. Winkler, tras sus lúcidos juicios sobre el pintor dados a conocer en 192429.

Ya en 1961, G. Marlier consagró al Maestro un importante y amplio trabajo en el que aduce sus razones para conservar, al excelente artista. el nombre provisional de «Maestro del Hijo Pródigo" hasta que nuevos descubrimientos puedan permitir una documentada identificación, y también llega a una serie de conclusiones, en las que trata de fijar sus caracteres artísticos a través del grupo de cuadros que estudia 30.

Entre nosotros, en 1954, Jesús Hernández Perera dedica al pintor un primer artículo a propósito de obras, existentes en España, que identifica como salidas del pincel del Maestro y, en 1957, hace nuevas aportaciones sobre el tema ${ }^{31-32}$. Más recientemente. en 1980, 1981 y 1989, M. Díaz Padrón. escribió interesantes páginas, dedicadas al pintor, en las que reúne un buen número de cuadros, la mayoría inéditos, conservados unos en España y otros en colecciones o instituciones extranjeras que hasta las publicaciones de este autor, algunos figuraban con atribución errónea a otros pintores ${ }^{33}$. Sólo citamos los estudios más directamente relacionados con el «Maestro del Hijo Pródigo» aunque, alusiones al mismo, se encuentran dentro de publicaciones a propósito de la pintura, de los Países Bajos del siglo xvi.

Conviene. pues conservar el nombre provisional de «Maestro del Hijo Pródigo", para el autor de esta pintura y estudiar sus características en relación con las que G. Marlier y los autores ya citados, están de acuerdo en definir como típicas de la personalidad artística de este pintor

26 G. Hulin de Loo: Catálogo del Museo de Bellas Artes de Gante.

27 G. Marlier: "L'Atelier du Maître du Fils Prodigue», Jaarboeck Koninklijk Museum voor Schöne Kunsten.Amberes, 1971 pág. 79.

28 Grete Ring: «Der Meister das Verlorenem Schönes», Jahrbuch für Kunstwissenschaft, 1923, pág. 196.

29 F. Winkler: Die Altniederländische Malerei, 1924, pág. 298.

30 G Marlier: «L'Atelier du Maître... Jarboeck Koninklijk..., 1961.

31 Jesús Hernández Perera: «Una Virgen del Maestro del Hijo Pródigo», Archivo Español de Arte, 1954, pág. 154.

32 Ibidem: "Algo más sobre el Maestro del Hijo Pródigo", Archivo Español de Arte, 1957, pág. 139.

33 M. Díaz Padrón: «Nuevas pinturas identificadas del Maestro del Hijo Pródigo. Goya. Revista de Arete, núm. 159, 1980, págs. 130-139. Un tríptico inédito del Maestro del Hijo Prodigo en el Museo de Pontevedra. Boletín del Museo del Prado, 1981, t. II, pág. 5. "Algunas tablas del Maestro del Hijo Pródigo en colecciones extranjeras». Goya. Revista de Arte, núm. 210, Madrid, 1989, pág. 322. 


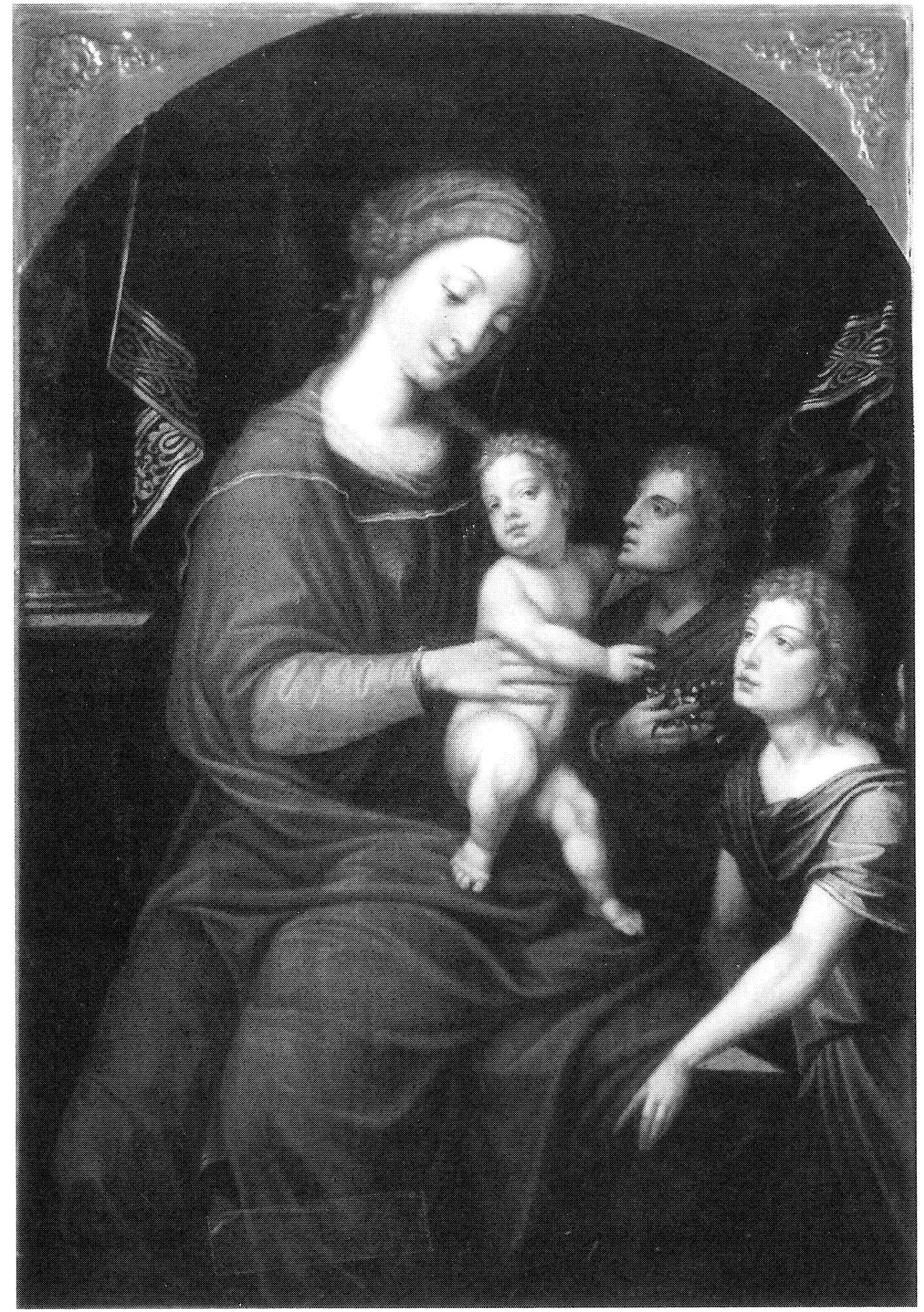

Fig. 18. "Maestro del Hijo Pródigo": Virgen con el Niño. Madrid. Colección particular 
del que todos suponen debió de formarse y trabajar en Amberes. La abundante producción que se le atribuye y las numerosas réplicas que existen de cuadros, agrupados dentro de su estilo hace suponer el funcionamiento de un floreciente taller comparable a los que mantenían Pieter Coecke, Pieter Aertsen, Frans Floris o Lambert Lombard que se cuentan entre los pintores de los Países Bajos, más importantes de la época. El hecho de no existir, que sepamos, ninguna composición idéntica a la de esta tabla, la convierte en una interesante aportación al catálogo de obras realizadas de propia mano del Maestro.

Dentro de las pinturas reconocidas, por los expertos, como del «Maestro del Hijo Pródigo» abundan las de tema religioso en dos variantes: cuadros de amplios paisajes con numerosos personajes a pequeña escala y otros en los que, el pintor. se siente atraído por mostrar a los protagonistas en primer plano, de figura entera o hasta poco más de las rodillas y ocupando la mayor parte del espacio pictórico, como es el caso de la tabla que nos ocupa.

En el cuadro, de colección madrileña, se advierten posibles fuentes, de inspiración compositiva, de gusto renacentista italiano y más concretamente, del llamado romanista que sigue esquemas empleados por Rafael. Así puede verse que las cuatro figuras en primer plano y vistas casi de cuerpo entero, forman un amplio grupo triangular, con vértice en la cabeza de la Virgen. También se inicia desde María una diagonal descendente, hacia el borde derecho, que establece un ritmo quebrado, entre los rostros de los personajes que intervienen. El escenario es un elegante interior con columna de jaspe de fuste rojizo y base bronceada que apoya sobre robusto pedestal de piedra e introduce una nota de riqueza. Desde la parte superior cae, en pliegues triangulares, un oscuro y lujoso cortinaje, con cenefa dorada, que contribuye a destacar la luminosa blancura de las carnaciones de la Virgen, el Niño y el ángel de primer término a lo que, también, ayuda el contraste con el rostro del personaje. que aparece entre los dos últimos, de tono más tostado o «bassanesco". El fondo oscuro resulta, además, ideal para realzar los elegantes y característicos tonos empleados, por el pintor, en las vestiduras.

No es clara la identificación del joven cubierto con túnica en castaño oscuro, al que el Niño distingue con gesto afectuoso, al apoyar sobre su pecho la mano derecha y rodearle el cuello con el brazo izquierdo. Podría representar a San Juan, como es frecuente en composiciones similares, flamencas e italianas de la época, pero en la iconografia, de este repetido tema, aunque hay ejemplos en los que se le pinta adolescente, es más habitual que se le figure en edad infantil al punto de que suele denominarse el asunto como "La Virgen, el Niño y San Juanito». Las, al parecer, florecillas que. el personaje sostiene en la mano a la altura del pecho. podrían aludir a algún otro Santo no reconocible porque por su colocación, no parece que intente representar a un posible donante.

Lo importante para nuestro propósito es, partiendo de la composición, formas y colores utilizados, en la pintura de colección madrileña, establecer las analogías, con otras obras, del "Maestro del Hijo Pródigo» al que aquí, consideramos autor de esta delicada tabla. La composición, de ascendencia italianizante, como hemos señalado, deriva, sin duda. de modelos rafaelescos que, el Maestro pudo conocer a través de los cuadros del genial italiano en, un posible viaje a Italia $\mathrm{o}$, más probablemente, inspirándose en los grabados de Marcantonio Raimondi que fue el gran difusor de la pintura de Rafael y cuyas estampas utilizaron, con frecuencia, los artistas de los Países Bajos. En el caso de nuestra tabla parece evidente su dependencia de la famosa Madonna de Foligno, de Rafael (Roma, Pinacoteca Vaticana) (Fig. 19). Es significativo, al respecto, comparar la actitud de los dos Niños que, al apoyar levemente los pies, sobre las rodillas de su Madre, adoptan una postura deslizante y con movimiento inestable, es decir, prácticamente, idéntica, en los dos cuadros. la variante se reduce a que en el del Vaticano, Jesús no mira hacia el frente, como en la pintura madrileña. Existe otro, grabado, de Marcantonio Raimondi, que muestra a la Virgen ante un fondo de cortinaje y similar inclinación de cabeza, con la 

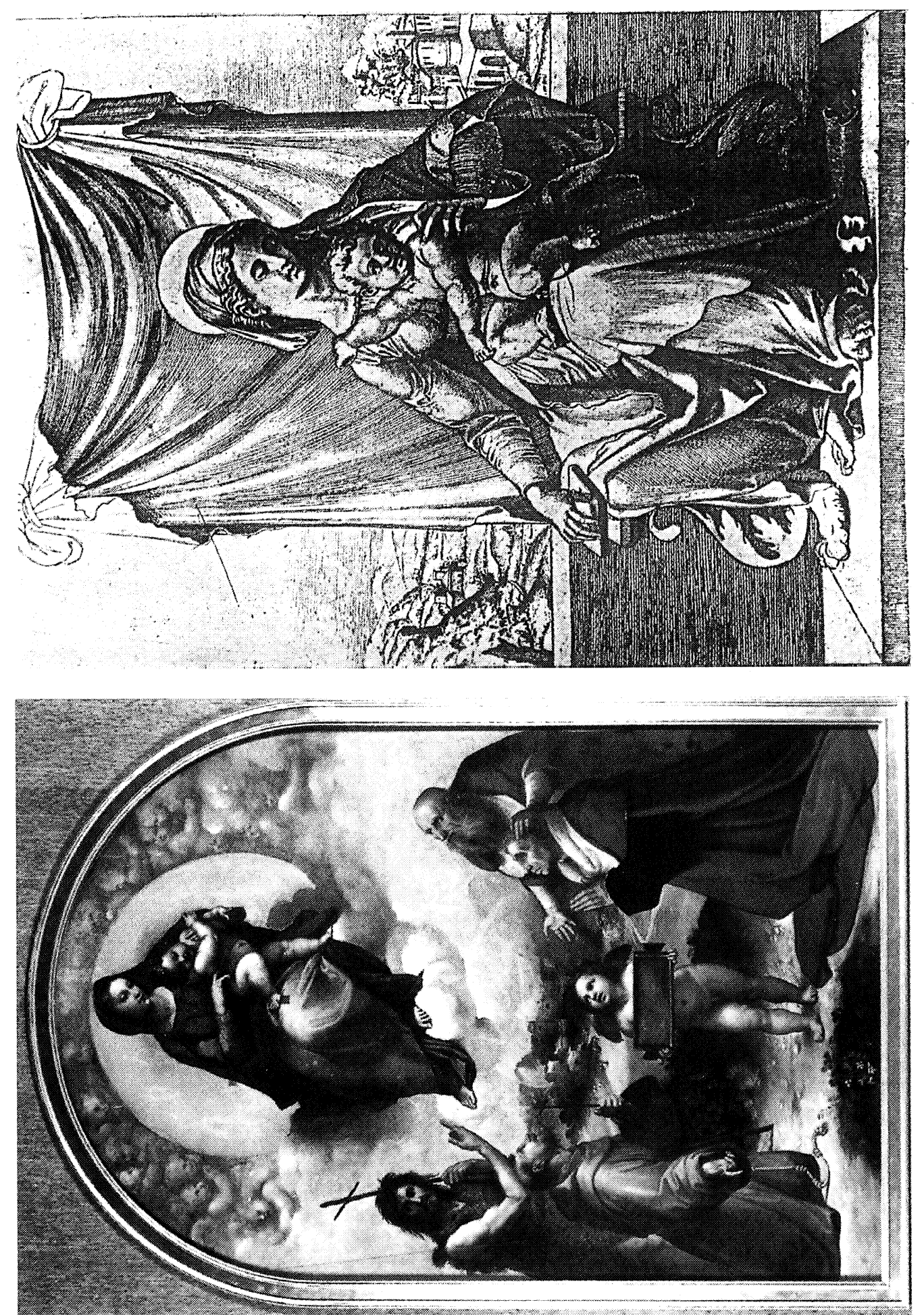

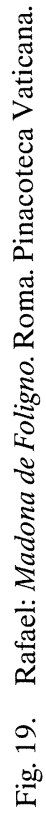


mirada baja, mientras que, el Niño dirige la suya al espectador como en el que aquí estudiamos ${ }^{34}$ (Fig. 20).

Los caracteres, que distinguen nuestra pintura, coinciden, en todo, con los reconocidos como más típicos del «Maestro del Hijo Pródigo». Seguimos a G. Marlier al comprobar que a pesar de las relaciones y paralelismos, con el arte de otros pintores, de la escuela de Amberes a los que ya hemos aludido «...las obras de este pintor sean tan fáciles de reconocer. Incluso cuando interpreta temas cuya invención remonta, posiblemente, a otros, él les impone un estilo, un colorido y unos rasgos que no podrían confundirse con los de ningún otro pintor", lo que, sin duda, denota su personalidad ${ }^{35}$. En los cuadros en los que, como en esta tabla, representa a los personajes en primor plano y casi de cuerpo entero, lo más significativo del estilo del Maestro son, quizás, sus rostros a los que personaliza una nariz larga, un tanto inclinada hacia la boca como se evidencia en la Virgen y en los vistos de perfil, de los que puede ser ejemplo el que está junto el Niño, muestran mentón prominente y afilado. Destacan, también, por su factura de modelado a base de sombras distribuidas con finas gradaciones que acentúan los pómulos y la musculatura en las figuras masculinas. y aun en el cuerpo del Niño, con recuerdo de la manera de hacer de Jan Gossaert y tal como aparece en buen número de Sagradas Familias atribuidas a Pieter Coecke. Las zonas luminosas, en cambio, se distinguen por un brillo con pulido casi marmóreo que aporta a sus pinturas una delicada belleza. Otro de sus rasgos típicos son las manos grandes en las que se aprecia, sin embargo, preocupación por conseguir elegancia como es visible en nuestro cuadro o en el, bien conocido, de Loth y sus hijas (Amberes, Musée Royal des Beaux Arts, n. ${ }^{\circ} 895$, T. $79 \times 110 \mathrm{~cm}$ ).

Una atención especial, merece el tratamiento de las telas, tanto por los colores que utiliza, como por la singular disposición de los plegados pues, en ambos casos, se manifiesta claramente la manera de hacer del «Maestro del Hijo Pródigo». Su gama cromática está dentro de la corriente manierista pero con inclinación por un variado y rico colorido en el que predominan los tonos rojos y verdes intensos: manto y túnica de 1a Virgen con matices asalmonados: manga de María y amarillentos: manteleta del ángel realizados, los dos últimos, con logradas calidades de seda. Esto añade, a sus pinturas, caracteres muy típicos que, unidos a los formales, más arriba citados, los hacen difíciles de confundir con los de ningún otro artista de su tiempo. En las telas se advierte una predilección por los pliegues amplios dispuestos en horizontal como se aprecia en el manto rojo de la Virgen o en la sedosa tela que cubre los hombros del ángel combinados, en éste, con otros angulosos que son igualmente, característicos del pintor.

El conjunto, de la tabla de Madrid, es un magnífico ejemplo de pintura romanista, inspirada en lo italiano que, sin embargo, no abandona, por la brillantez de colorido, factura y materia, su vinculación a las calidades de tradición flamenca.

Establecer una cronología dentro de la carrera artística de este Maestro, plantea un difícil problema que deriva de la falta de noticias concretas al no contar, hasta la fecha, con ninguna obra firmada con su desconocido nombre, ni existir datos de archivo que puedan aplicarse, con seguridad, para definir la personalidad del pintor. La conclusión a la que llega G. Marlier, para las obras que considera de la propia mano del Maestro, sería que «al lado de los talleres, tan prósperos de Pieter Coecke y Frans Floris, existía en Amberes un taller no menos floreciente dirigido por... el que se conoce, provisionalmente, como «Maestro de1 Hijo Pródigo» ${ }^{36}$. En todo caso dice que sus pinturas dan perfecta idea del gusto artístico que reinaba, en los Países Bajos del Sur, hacia mediados del siglo XVI y poco más se puede añadir al respecto, aunque el propio estilo del cuadro, que damos a conocer, apunta hacia una fecha aproximada ca. 1550-1560.

34 Rafaello in Vaticano (Electa). Milán, 1984, págs. 267-270. Bartsch, vol. 26, Marcantonio Raimondi. Nueva York, 1978, núm. 52, fig. 46. P. L. De Vacchi (para Madonna de Foligno). Florencia, 1984, lám. CXIII.

35 G. Marlier: "L'Atelier du Maître...», Jaarboeck Koninklijk..., 1961.

36 Ibidem. 